\title{
Non-small cell lung cancer PC-9 cells exhibit increased sensitivity to gemcitabine and vinorelbine upon acquiring resistance to EGFR-tyrosine kinase inhibitors
}

\author{
JUNKO HAMAMOTO $^{1 *}$, HIROYUKI YASUDA ${ }^{1}$, KAITO AIZAWA $^{2 *}$, \\ MAKOTO NISHINO $^{1}$, SHIGENARI NUKAGA ${ }^{1}$, TOSHIYUKI HIRANO ${ }^{1}$, ICHIRO KAWADA ${ }^{1}$, \\ KATSUHIKO NAOKI $^{3}$, TOMOKO BETSUYAKU ${ }^{1}$ and KENZO SOEJIMA ${ }^{1}$
}

${ }^{1}$ Division of Pulmonary Medicine, Department of Medicine, Keio University School of Medicine; ${ }^{2}$ Keio University School of Medicine, Tokyo 160-8582; ${ }^{3}$ Keio Cancer Center, Keio University Hospital, Tokyo 160-0016, Japan

Received July 1, 2016; Accepted March 21, 2017

DOI: $10.3892 / \mathrm{ol} .2017 .6591$

\begin{abstract}
Epidermal growth factor receptor (EGFR)-tyrosine kinase inhibitors (EGFR-TKIs) are widely used for the treatment of non-small cell lung cancers (NSCLCs) harboring EGFR-activating mutations. However, lung cancer cells inevitably acquire resistance to these EGFR-TKIs. The majority of patients whose lung cancer acquires resistance to EGFR-TKIs are subjected to treatment using cytotoxic agents. The present study aimed to determine if lung cancer cells acquiring resistance to EGFR-TKIs also develop altered sensitivity to cytotoxic agents. It was revealed that lung cancer cells that had developed resistance to EGFR-TKIs had increased sensitivity to gemcitabine and vinorelbine compared with EGFR-TKI naïve cells. The expression levels of ATP-binding cassette (ABC) transporter genes, including $A B C C 3, A B C C 5$ and $A B C G 2$, were observed to be commonly repressed in EGFR-TKI-resistant cells. In addition, two cases were identified in which gemcitabine and vinorelbine exerted marked responses to lung cancers that had acquired resistance to EGFR-TKIs, even with late-line treatment. Therefore, it was proposed that gemcitabine and vinorelbine may be effective agents for patients with lung cancer previously treated with EGFR-TKIs.
\end{abstract}

Correspondence to: Dr Hiroyuki Yasuda, Division of Pulmonary Medicine, Department of Medicine, Keio University School of Medicine, 35 Shinanomachi, Shinjuku, Tokyo 160-8582, Japan E-mail: hiroyukiyasuda@a8.keio.jp

*Contributed equally

Abbreviations: EGFR, epidermal growth factor receptor; TKIs, tyrosine kinase inhibitors; NSCLC, non-small cell lung cancer; ER, erlotinib-resistant; GR, gefinitib-resistant; ABC, adenosine triphosphate-binding cassette

Key words: lung cancer, gemcitabine, vinorelbine, epidermal growth factor receptor, tyrosine kinase inhibitors, resistance

\section{Introduction}

Lung cancer is the leading cause of cancer-associated mortality worldwide (1). Epidermal growth factor receptor (EGFR) mutations have been identified in $10-30 \%$ of non-small cell lung cancers (NSCLCs) $(2,3)$. EGFR-tyrosine kinase inhibitors (TKIs) have been developed to target mutated EGFR (4). The first-generation EGFR-TKIs, namely gefitinib and erlotinib, which bind reversibly to the adenosine triphosphate (ATP)-binding site of the receptor, markedly changed the treatment strategy for patients harboring EGFR-mutated lung cancers (4). The response rates to gefitinib or erlotinib are $60-80 \%(3,5)$. Significant benefits of gefitinib or erlotinib treatment in patients with NSCLC harboring EGFR-TKI-sensitizing mutations have been repeatedly demonstrated in multiple clinical trials $(6,7)$. However, despite the initial favorable response, lung cancer cells eventually acquire resistance to gefitinib or erlotinib (4). Studies from the last few years have identified several EGFR-TKI resistance mechanisms (8-12). The main mechanism, accounting for $\sim 50 \%$ of the resistance, is a secondary mutation in the EGFR gene subsequent to the initial TKI-sensitizing mutations, specifically T790M in exon 20 of EGFR (13,14). The other mechanisms include amplification of the $M E T$ oncogene $(10,15,16)$, activation of the hepatocyte growth factor (HGF)-MET signaling pathway through HGF overexpression (17), epithelial-mesenchymal transition (18), EGFR amplification transformation to SCLC (19) and activation of the fibroblastic growth factor (FGF) 2-FGF receptor (FGFR) 1 signaling pathway through an autocrine loop (11).

In general, lung cancer cells acquire resistance to EGFR-TKIs in $\sim 1$ year (8). For patients whose lung cancer acquires resistance to initially administered EGFR-TKIs, multiple cytotoxic agents are available, including cisplatin, carboplatin, docetaxel, paclitaxel, irinotecan, gemcitabine, vinorelbine and pemetrexed (20). Despite the hematological and non-hematological toxicity of these cytotoxic agents, their efficacy has been consistently reported in multiple settings (21-25). However, whether lung cancer cells that have acquired resistance to EGFR-TKIs also exhibit altered sensitivity to cytotoxic agents remains to be ascertained. An 
alternative mechanism for resistance to EGFR-TKIs may be through the upregulation of resistance-associated genes, including ATP-binding cassette (ABC) transporter family genes. ABC proteins contribute to chemoresistance through the efflux of anticancer drugs from cancer cells (26). The association between ABC expression and EGFR-TKI resistance has yet to be clarified. The present study attempted to explore the response of EGFR-TKI-resistant lung cancer cells to cytotoxic agents.

\section{Materials and methods}

Cell lines. PC-9 cells were a kind gift from Dr Susumu Kobayashi (Beth Israel Deaconess Medical Center, Boston, MA, USA). EGFR-TKI-resistant cell lines were previously established by long-term ( $\sim 6$ months) exposure to erlotinib and gefitinib in our previous study (19). Erlotinib-resistant PC-9 (PC-9ER) cells arose following chronic exposure to erlotinib through the acquisition of the secondary EGFR mutation, T790M. Gefitinib-resistant PC-9 (PC-9GR) cells were obtained by chronic exposure to gefitinib through activation of the FGF2-FGFR1 signaling pathway (11). The PC-9, PC-9ER and PC-9GR cells were cultured in RPMI-1640 growth medium supplemented with $10 \%$ fetal bovine serum (Gibco; Thermo Fisher Scientific, Inc., Waltham, MA, USA) at $37^{\circ} \mathrm{C}$ in a humidified $5 \% \mathrm{CO}_{2}$ incubator. The present study was approved by the Ethics Committee of Keio University, School of Medicine (Tokyo, Japan).

Materials. Cisplatin and docetaxel were purchased from Wako Pure Chemical Industries, Ltd. (Osaka, Japan). Gemcitabine and vinorelbine were purchased from Sigma-Aldrich (Merck KGaA, Darmstadt, Germany). Pemetrexed was purchased from LC Laboratories (Woburn, MA, USA).

Cell proliferation assay. A proliferation assay was performed on PC-9, PC-9ER and PC-9GR cells seeded on 96-well plates at a density of 2,000 cells/50 $\mu 1$ medium/well, which were incubated as previously described. The following day, $50 \mu \mathrm{l}$ of RPMI-1640 medium containing each anticancer chemotherapy drug: Cisplatin, docetaxel, pemetrexed, gemcitabine or vinorelbine, dissolved in dimethyl sulfoxide (DMSO) to a concentration of $0.0001,0.001,0.01,0.1,1$ or $10 \mu \mathrm{M}$, was added to each well. Control cells were treated with DMSO. After $72 \mathrm{~h}$ of incubation as previously described, the cells were treated with the CellTiter $96{ }^{\circledR}$ AQueous One Solution Cell Proliferation Assay kit (Promega Corporation, Madison, WI, USA), according to the manufacturer's protocol. Cell population density was then measured as $490 \mathrm{~nm}$ absorbance using a microplate reader (Model 680; Bio-Rad Laboratories, Inc., Hercules, CA, USA).

Apoptosis assay. A total of $3 \times 10^{4}$ PC-9 and PC-9 ER cells were seeded into each well of 6 -well plates. The cells were treated with $3 \mu \mathrm{M}$ gemcitabine or $0.1 \mu \mathrm{M}$ vinorelbine, for $48 \mathrm{~h}$ in the previously described conditions. Control cells were treated with the same concentration of DMSO. The apoptotic cells were stained using the TACS Annexin V-FITC Apoptosis Detection kit (R\&D Systems, Inc., Minneapolis, MN, USA), which included propidium iodide, according to the manufacturer's protocol. The proportion of apoptotic cells was evaluated using the Gallios Flow Cytometer (Beckman Coulter, Inc., Brea, CA, USA) and analyzed with FlowJo software 7.6.5 (TOMY Digital Biology Co., Ltd., Tokyo, Japan).

Reverse transcription-quantitative polymerase chain reaction $(R T-q P C R)$. Total RNA was isolated from cells using an RNeasy Mini kit (Qiagen, Inc., Valencia, CA, USA) according to the manufacturer's protocol, and $2 \mu \mathrm{g}$ of total RNA was subjected to RT using the High-Capacity RNA-to-cDNA kit (Thermo Fisher Scientific, Inc., Waltham, MA, USA) according to the manufacturer's protocol. qPCR was performed using the KAPA SYBR FAST qPCR kit (Kapa Biosystems, Inc., Wilmington, MA, USA) and an ABI Prism 7000 Sequence Detection System (Applied Biosystems; Thermo Fisher Scientific, Inc.) according to the manufacturer's protocol. The thermocycler settings were 40 cycles of $95^{\circ} \mathrm{C}$ for $5 \mathrm{sec}$ and $60^{\circ} \mathrm{C}$ for $30 \mathrm{sec}$. The sequences of the primers used in the present study were bought from the Roche Life Science Assay Design Center (Roche Molecular Systems, Inc., Pleasanton, CA, USA), and were as follows: GAPDH forward, 5'-GAGTCAACGGAT TTGGTCGT-3' and reverse, 5'-TTGATTTTGGAGGGATCT CG-3'; ATP-binding cassette $(A B C) B 1$ forward, 5'-GAAATT TAGAAGATCTGATGTCAAACA-3' and reverse, 5'-ACT GTAATAATAGGCATACCTGGTCA-3'; ABCC1 forward, 5'-CCATGTGGGAAAACACATCTT-3' and reverse, 5'-CTG TGCGTGACCAAGATCC-3'; ABCC2 forward, 5'-AGTGAA TGACATCTTCACGTTTG-3' and reverse, 5'-CTTGCAAAG GAGATCAGCAA-3'; ABCC3 forward, 5'-CCTGGCTGTGCTCTACACCT-3' and reverse, 5'-GATTCCAGCCGCTTC AGTT-3'; ABCC5 forward, 5'-GCAGTAAAGCCAGAGGAA GG-3' and reverse, 5'-CAGCCTGGATGTAGACACCATA-3'; and $A B C G 2$ forward, 5'-TGGCTTAGACTCAAGCACAGC-3' and reverse, 5'-TCGTCCCTGCTTAGACATCC-3'.

Samples were analyzed in triplicate, and the experiment was repeated three times. Relative quantification values were calculated by comparison with GAPDH using the quantification cycle $(\mathrm{Cq})$ method (27).

Patients and treatments. The patient records of two individuals treated for EGFR mutation-positive NSCLC at Keio University hospital were selected based on a case review conference, in which patients with lung cancer who had experienced extraordinary or unusual patterns of disease presentation or progression, or patients who received unusual treatment courses, were discussed. Informed written consent was obtained from these individuals.

Statistical analysis. Data are presented as mean \pm standard deviation. Statistical analysis for the proliferation assay and RT-qPCR was performed using GraphPad Prism software, version 4.0 (GraphPad Software, Inc., La Jolla, CA, USA). Two-sided Student's t tests were used for comparisons. $\mathrm{P}<0.05$ was considered to indicate a statistically significant difference.

\section{Results}

Increased sensitivity of EGFR-TKI-resistant cells to gemcitabine and vinorelbine. PC-9 cells harboring an $E G F R$-activating mutation (EGFR exon 19 deletion) (28) were 


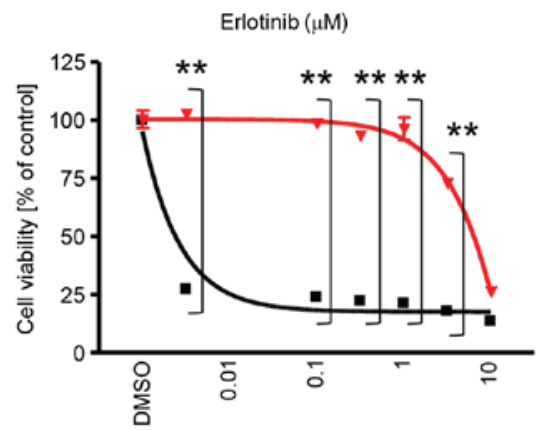

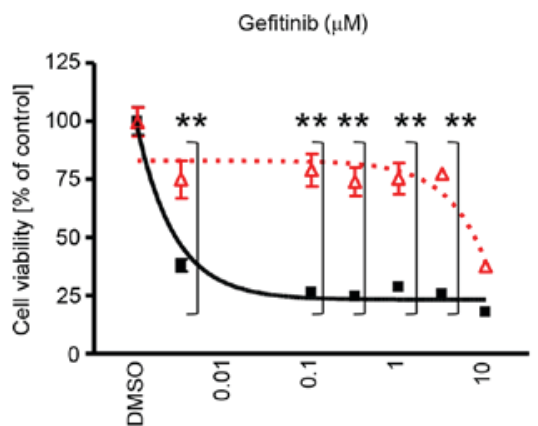

B
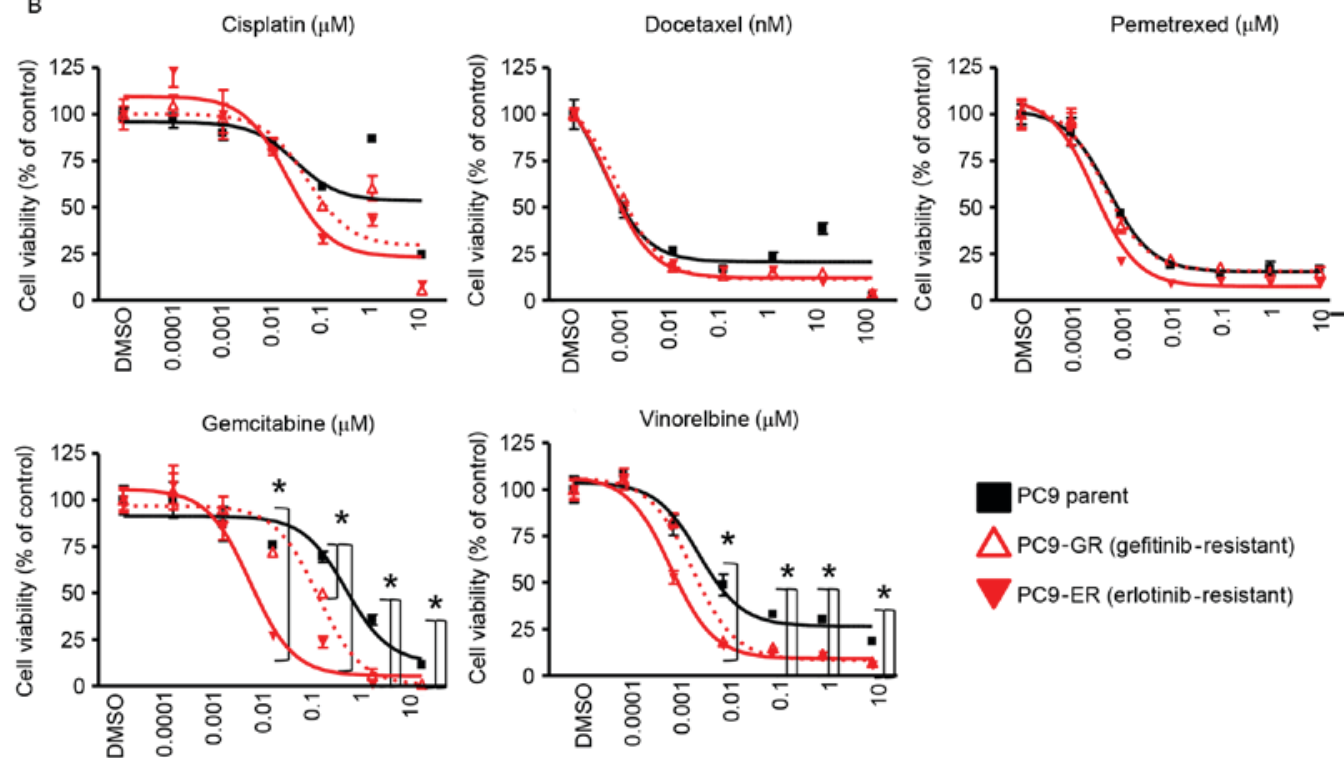

PC9 parent

$\triangle \mathrm{PC9}-\mathrm{GR}$ (gefitinib-resistant)

7 PC9-ER (erlotinib-resistant)

Figure 1. Sensitivity of epidermal growth factor receptor-tyrosine kinase inhibitor-sensitive and resistant cell lines to cytotoxic agents. Results of MTS assay for PC-9, PC-9GR and PC-9ER cells. The proportional cell viability is shown. (A) Erlotinib, gefitinib, (B) cisplatin, docetaxel, pemetrexed, gemcitabine and vinorelbine were used as cytotoxic agents. Error bars indicate standard deviation. ${ }^{*} \mathrm{P}<0.05 ;{ }^{* *} \mathrm{P}<0.01$. DMSO, dimethyl sulfoxide; GR, gefitinib-resistant; ER, erlotinib-resistant.

used in the present study (29). The established EGFR-TKI-resistant cells used in the present study were designated as PC-9ER and PC-9GR cells. PC-9ER cells became resistant to erlotinib by acquiring the EGFR T790M gatekeeper mutation (i.e., a mutation that sterically hinders inhibitor interaction), whereas PC-9GR cells became resistant to gefitinib through activation of the FGF2-FGFR1 pathway (11). First, the resistance of PC-9ER and PC-9GR cells to EGFR-TKIs was confirmed. The proliferation of PC-9 parent cells was inhibited by erlotinib and gefitinib at $0.01 \mu \mathrm{M}$; however, the proliferation of PC-9ER and PC-9GR was not significantly inhibited by even $3 \mathrm{mM}$ erlotinib or gefitinib (Fig. 1A). To examine whether EGFR-TKI-resistant cells exhibit altered sensitivity to cytotoxic agents, the sensitivity of PC-9, PC-9ER and PC-9GR cells was evaluated by MTS assay with or without cytotoxic agents. The cytotoxic agents included in the present study were cisplatin, docetaxel, pemetrexed, gemcitabine and vinorelbine, all of which are widely used in the clinic for the treatment of patients with NSCLC $(22,25,30)$. The sensitivity of EGFR-TKI-resistant cells to cisplatin, docetaxel and pemetrexed was comparable with that of PC-9 parent cells. By contrast, increased sensitivity of EGFR-TKI-resistant cells to gemcitabine and vinorelbine was observed (Fig. 1B). These results indicated that EGFR-TKI-resistant cells had increased sensitivity to gemcitabine and vinorelbine.

Increased apoptosis of EGFR-TKI-resistant cells in response to gemcitabine and vinorelbine. Cytotoxic agents damage the DNA of cancer cells, which subsequently induces cancer cell apoptosis (31). The aforementioned findings prompted the authors of the present study to examine whether EGFR-TKI-resistant cells are more prone to undergo apoptosis with gemcitabine and vinorelbine treatment. The apoptosis of cancer cells was examined using Annexin $\mathrm{V}$ and propidium iodide staining. The proportions of Annexin V-positive PC-9 cells treated with gemcitabine and vinorelbine were 13.6 and $24.2 \%$, respectively, while the proportions of Annexin-V-positive PC-9ER cells treated with gemcitabine and vinorelbine were 29.9 and $53.5 \%$, respectively (Fig. 2). These data indicated that EGFR-TKI-resistant cells underwent increased apoptosis upon gemcitabine and vinorelbine treatment.

Increased or decreased expression of $A B C$ transporters in EGFR-TKI-resistant cells. ABC transporters efflux cytotoxic agents out from cancer cells, thereby contributing to the insensitivity of cancer cells to cytotoxic agents $(26,32)$. 


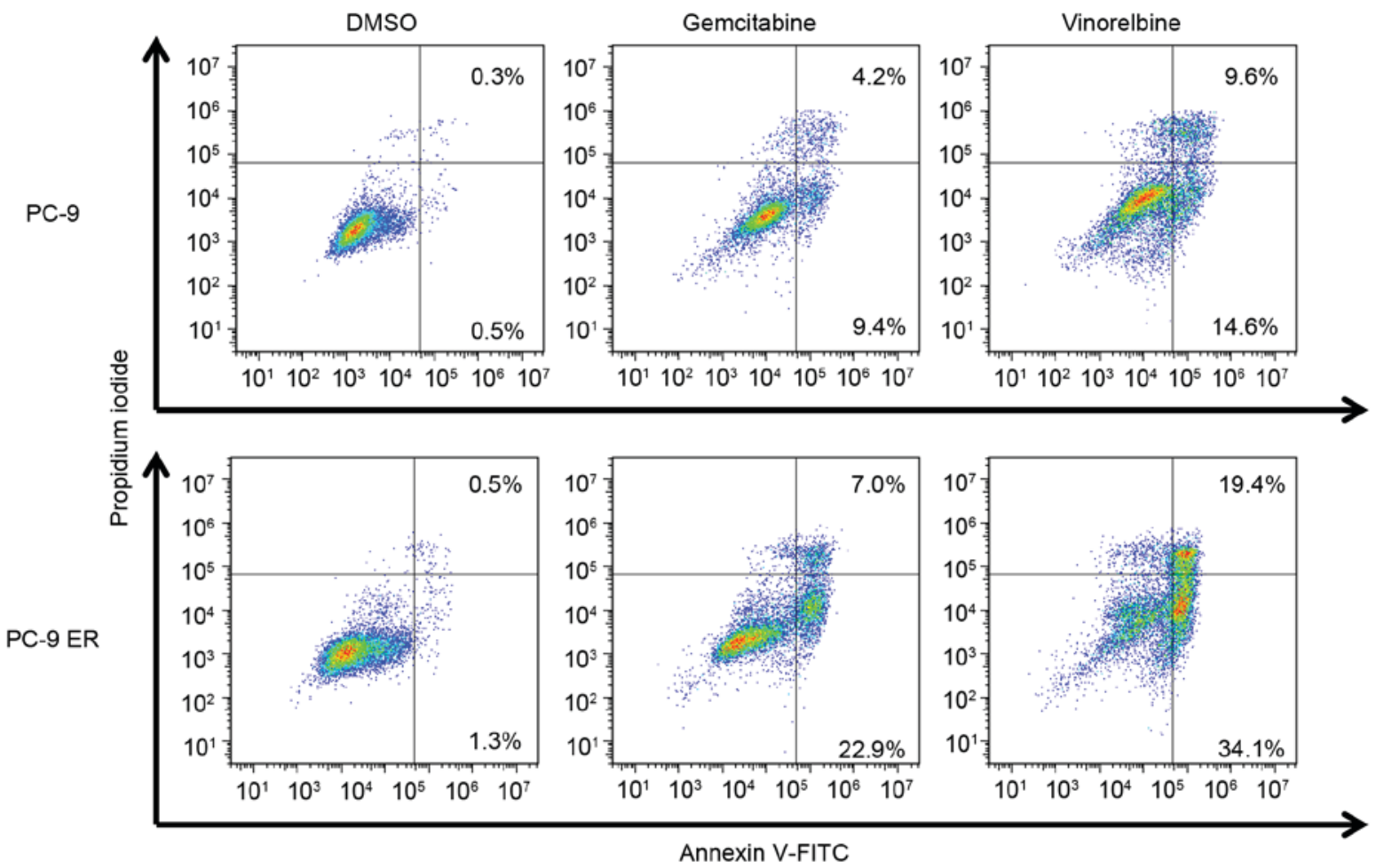

Figure 2. Apoptosis analysis for PC-9 and PC-9ER cells subjected to gemcitabine and vinorelbine treatment. Results of the apoptosis assay using cytometry. PC-9 or PC-9ER cells were treated with gemcitabine or vinorelbine for $48 \mathrm{~h}$. Subsequently, the cells were stained with propidium iodide and Annexin V-FITC The numbers indicate the proportion of Annexin V-positive and/or propidium iodide-positive cells. DMSO, dimethyl sulfoxide; FITC, fluorescein isothiocyanate; ER, erlotinib-resistant.
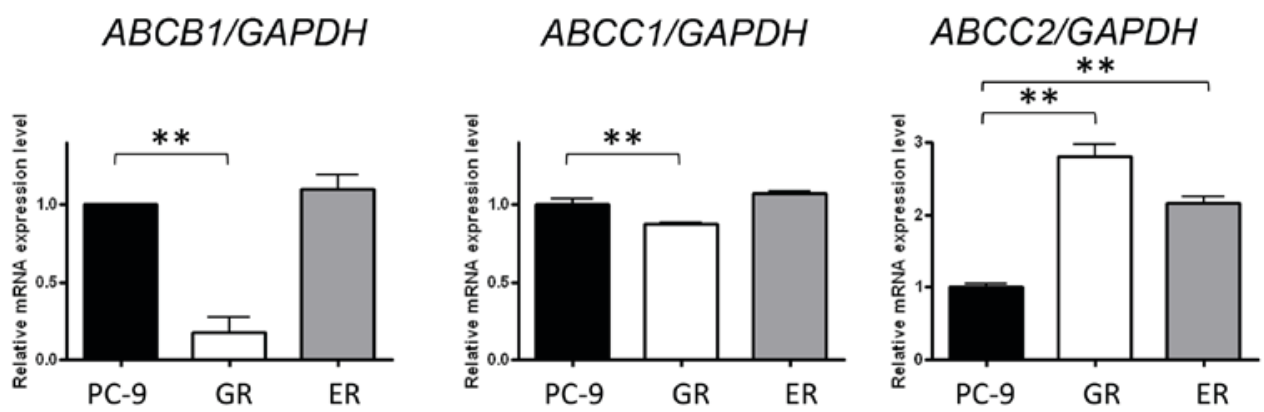

$A B C C 3 / G A P D H$

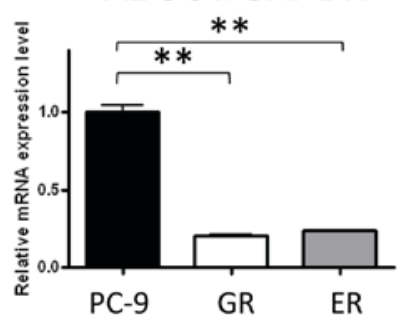

$A B C C 5 / G A P D H$
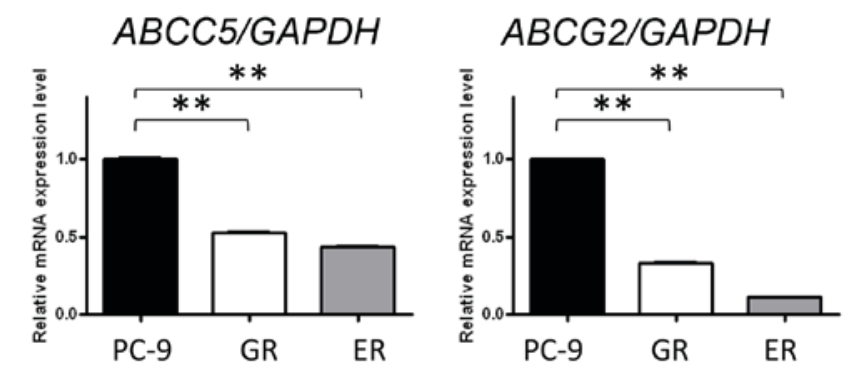

$P C-9$ parent

GR (PC-9 Gefitinib resistant)

ER (PC-9 Erlotinib resistant)

Figure 3. Expression of ABC transporter genes in epidermal growth factor receptor-tyrosine kinase inhibitor-sensitive and resistant cell lines. Expression of the indicated genes is shown for PC-9, PC-9ER and PC-9GR cells relative to the expression of GAPDH. Error bars indicate standard deviation. ${ }^{* *} \mathrm{P}<0.01$. ABC, ATP-binding cassette; ER, erlotinib-resistant; GR, gefinitib-resistant; mRNA, messenger RNA.

Certain ABC transporter family members have been reported to act as determinants of cell sensitivity to gemcitabine and vinorelbine (33). The expression levels of ABC (34) transporters, including $A B C B 1, A B C C 1, A B C C 2, A B C C 3$, $A B C C 5$ and $A B C G$, were evaluated. $A B C B 1$ and $A B C C 1$ were only significantly reduced in PC-9GR cells, whereas $A B C C 2$ 


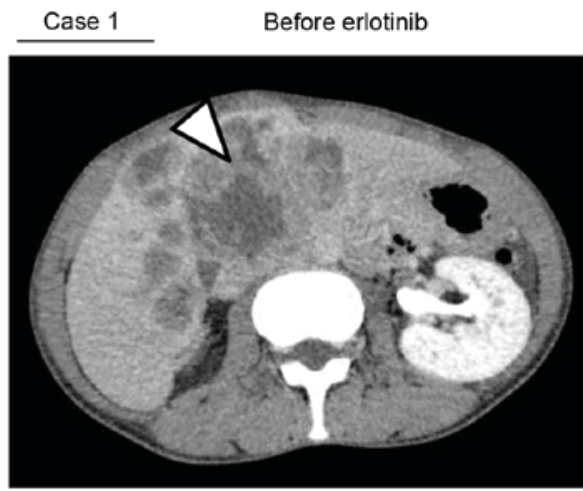

C

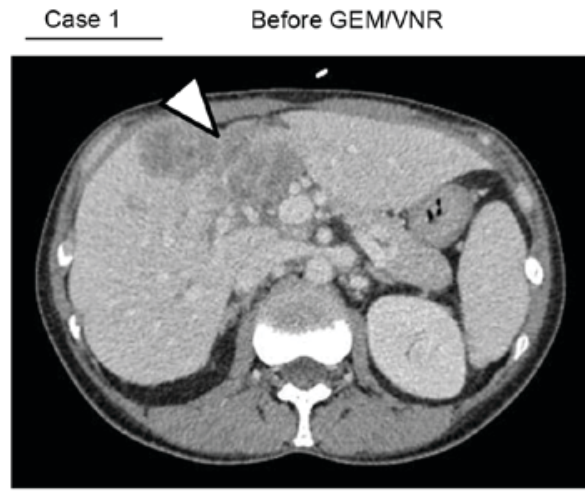

E

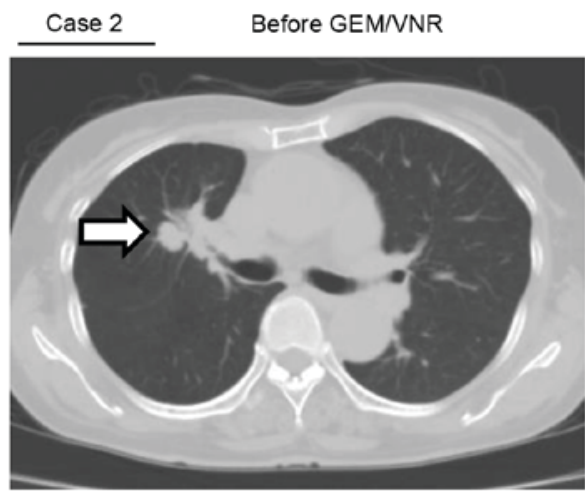

B

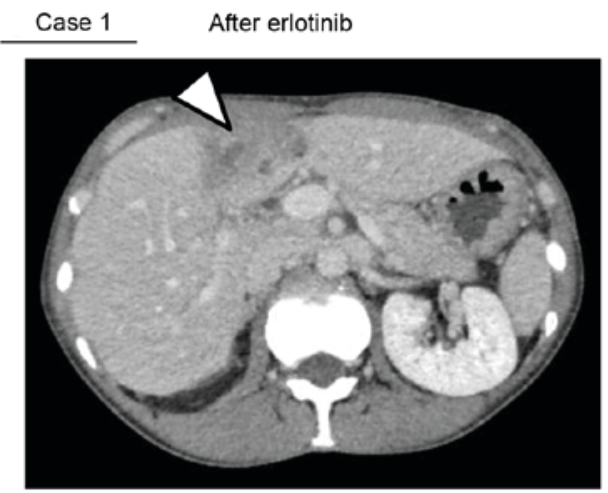

D

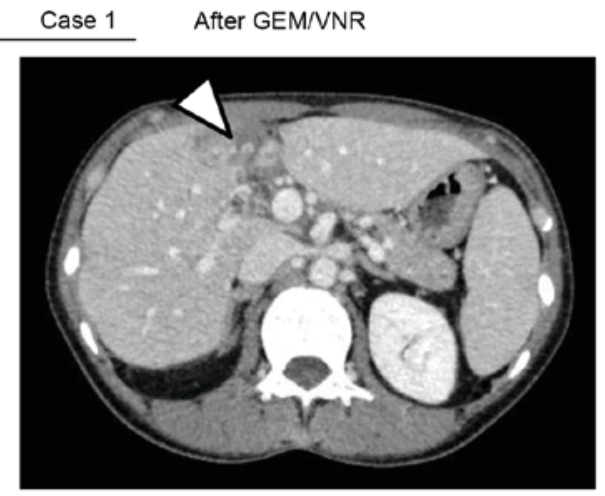

$F$

Figure 4. CT scan images of two patients with epidermal growth factor receptor mutation-positive lung cancer. CT scan images of patient 1 (A) prior to erlotinib, (B) following erlotinib, (C) prior to GEM/VNR and (D) following GEM/VNR treatment. CT scan images of patient 2 (E) prior to GEM/VNR and (F) following GEM/VNR treatment. White arrows indicate the tumors in each image. CT, computed tomography; GEM, gemcitabine; VNR, vinorelbine.

expression was significantly upregulated and $A B C C 3, A B C C 5$ and $A B C G 2$ were significantly repressed in PC-9GR and PC-9ER cells compared with PC-9 cells (Fig. 3). These data indicated that the decreased expression of $A B C C 2, A B C C 3$, $A B C C 5$ and $A B C G 2$ may be associated with increased sensitivity of EGFR-TKI-resistant cells to gemcitabine and vinorelbine.

Clinical cases. It was then examined whether the acquisition of sensitivity to gemcitabine or vinorelbine, as demonstrated by the present study in vitro, occurred in a clinical setting. Two notable cases were identified, which are described below.
Patient 1. The first patient was a 48-year-old non-smoking woman diagnosed with EGFR mutation-positive (exon 19 deletion) lung adenocarcinoma by bronchoscopy in September 2014 at Keio University hospital. A subsequent positron emission tomography scan revealed liver and bone metastatic lesions (cT4N3M1b, stage IV). Erlotinib (150 mg) was administered as the first-line therapeutic against the disease, and it controlled the lesions for 195 days (Fig. 4A and B). Next, 6 courses of carboplatin (area under curve $=5)$, pemetrexed $\left(500 \mathrm{mg} / \mathrm{m}^{2}\right)$ and bevacizumab $(15 \mathrm{mg} / \mathrm{kg})$ were administered as the second-line treatment, which was followed by 3 courses of pemetrexed as a maintenance therapy until the progression of the disease, including liver metastatic lesions. The patient is currently 
being treated with a regimen of gemcitabine $\left(1,000 \mathrm{mg} / \mathrm{m}^{2}\right)$ plus vinorelbine $\left(25 \mathrm{mg} / \mathrm{m}^{2}\right)$ for 9 courses. Notably, despite the late-line chemotherapy, the regimen was effective, and it continues to confer sufficient antineoplastic effect with tolerable side effects (Fig. 4C and D). The patient remains alive at the time of publishing.

Patient 2. The second patient was a 75-year-old woman, who was diagnosed with EGFR mutation-positive (exon 21 L858R) lung adenocarcinoma by bronchoscopy in May 2007 at Keio University hospital. Subsequent computed tomography and magnetic resonance imaging scans revealed the staging as cT4N2M0, stage IIIB. Gefitinib (250 mg) was the first-line regimen administered to the patient, and this EGFR-TKI stabilized the primary lesions for 14 months. Subsequent to confirming the enlargement of the primary lesion, 4 courses of carboplatin plus docetaxel $\left(60 \mathrm{mg} / \mathrm{m}^{2}\right)$ were administered as second-line chemotherapy. Brain and hilar mediastinal lymph node metastases was observed after 11 months. The third-line option was to re-challenge the brain lesions with gefitinib $(250 \mathrm{mg})$ and $\gamma$-knife radiosurgery. After 6 months of gefitinib treatment, the primary lesion again progressed; therefore, fourth-line chemotherapy was administered, using gemcitabine $\left(1,000 \mathrm{mg} / \mathrm{m}^{2}\right)$ plus vinorelbine $\left(25 \mathrm{mg} / \mathrm{m}^{2}\right)$ for 7 courses. The late-line non-platinum doublet chemotherapy regimen markedly led to complete remission of advanced lung adenocarcinoma in this case (Fig. 4E and F). In addition, following the re-growth of the primary lesion 1 year after being treatment-free, the re-challenge of 4 courses of gemcitabine plus vinorelbine controlled the lesions. The patient remains alive at the time of publishing.

Clinical cases conclusion. These results indicated the possibility of using gemcitabine and vinorelbine as effective agents for patients whose lung cancer acquires resistance to EGFR-TKIs.

\section{Discussion}

In the present study, the sensitivity of EGFR-TKI-resistant cells to five cytotoxic agents commonly used in the treatment of patients with NSCLC was evaluated. Notably, an increased sensitivity of EGFR-TKI-resistant cells to gemcitabine and vinorelbine was observed.

Several factors are reported to affect cell sensitivity to gemcitabine and vinorelbine. Thus, overexpression of breast cancer gene 1 (30), class III $\beta$-tubulin (35), the ABC transporter family genes $A B C B 1 /$ multidrug resistance protein 1 (MDR1) and ABCC10/multidrug resistance-associated protein 71 (33), and the non-ABC transporter protein Ral interacting protein 76 (36), has been reported to lead to resistance to vinorelbine. Overexpression of ABCC5 (34), human equilibrative nucleoside transporter 1 (37) and ribonucleotide reductase (38) has been reported to lead to resistance to gemcitabine. Several members of the ABC transporter superfamily, including $M D R 1$ (also known as $A B C C 1$ ), confer drug resistance to drug-sensitive cells by effluxing anticancer or antiviral agents or their metabolites from cells when expressed at high levels (32). Therefore, the gene expression levels of the $\mathrm{ABC}$ transporter family in relation to gemcitabine/vinorelbine treatment in the three tested cell lines were examined. It was observed that the expression levels of $A B C C 3, A B C C 5$ and $A B C G 2$ were commonly repressed in EGFR-TKI-resistant cell lines, similar to an earlier study demonstrating the association between $A B C C 5$ expression levels and acquired resistance to gemcitabine (34). However, whether the repressed expression of $A B C C 3, A B C C 5$ and $A B C G 2$ affects the sensitivity to gemcitabine and vinorelbine of EGFR-TKI-resistant cells is not known. To understand the mechanism of increased sensitivity to gemcitabine and vinorelbine of EGFR-TKI-resistant cells, additional in vitro and in vivo experiments are necessary.

In the two cases reported in the present study, gemcitabine and vinorelbine exerted a marked response towards lung cancers that had acquired resistance to EGFR-TKIs, even with late-line treatment. However, it remains unclear whether previous EGFR-TKI treatment increased the sensitivity to subsequent gemcitabine and vinorelbine treatment. This aspect requires further investigation in future studies.

The present findings indicated a new treatment option for EGFR-TKI-resistant NSCLC chemotherapy. However, additional evaluation through randomized trials remains necessary.

\section{Acknowledgements}

The authors would like to thank Ms. Mikiko Shibuya (Division of Pulmonary Medicine, Department of Medicine, Keio University School of Medicine) for her technical assistance. The present study was supported in part by Grants-in-Aid for Scientific Research on Priority Areas from the Ministry of Education, Culture, Sports, Science and Technology of Japan awarded to K. Soejima (grant no. 22590870) and H. Yasuda (grant nos. 25860656, 15H05666 and 15K14398).

\section{References}

1. Siegel R, Naishadham D and Jemal A: Cancer statistics, 2013. CA Cancer J Clin 63: 11-30, 2013.

2. Cancer Genome Atlas Research Network: Comprehensive molecular profiling of lung adenocarcinoma. Nature 511: 543-550, 2014

3. Jänne PA and Johnson BE: Effect of epidermal growth factor receptor tyrosine kinase domain mutations on the outcome of patients with non-small cell lung cancer treated with epidermal growth factor receptor tyrosine kinase inhibitors. Clin Cancer Res 12: 4416s-4420s, 2006.

4. Zhou C and Yao LD: Strategies to improve outcomes of patients with Egrf-Mutant non-small cell lung cancer: Review of the literature. J Thorac Oncol 11: 174-186, 2016.

5. Mitsudomi T and Yatabe Y: Mutations of the epidermal growth factor receptor gene and related genes as determinants of epidermal growth factor receptor tyrosine kinase inhibitors sensitivity in lung cancer. Cancer Sci 98: 1817-1824, 2007.

6. Shepherd FA, Rodrigues Pereira J, Ciuleanu T, Tan EH, Hirsh V, Thongprasert S, Campos D, Maoleekoonpiroj S, Smylie M, Martins R, et al: Erlotinib in previously treated non-small-cell lung cancer. N Engl J Med 353: 123-132, 2005.

7. Mok TS, Wu YL, Thongprasert S, Yang CH, Chu DT, Saijo N, Sunpaweravong P, Han B, Margono B, Ichinose Y, et al: Gefitinib or carboplatin-paclitaxel in pulmonary adenocarcinoma. N Engl J Med 361: 947-957, 2009.

8. Morgillo F, Della Corte CM, Fasano M and Ciardiello F: Mechanisms of resistance to EGFR-targeted drugs: Lung cancer. ESMO Open 1: e000060, 2016.

9. Kobayashi S, Ji H, Yuza Y, Meyerson M, Wong KK, Tenen DG and Halmos B: An alternative inhibitor overcomes resistance caused by a mutation of the epidermal growth factor receptor. Cancer Res 65: 7096-7101, 2005. 
10. Engelman JA, Zejnullahu K, Mitsudomi T, Song Y, Hyland C, Park JO, Lindeman N, Gale CM, Zhao X, Christensen J, et al: MET amplification leads to gefitinib resistance in lung cancer by activating ERBB3 signaling. Science 316: 1039-1043, 2007.

11. Terai H, Soejima K, Yasuda H, Nakayama S, Hamamoto J, Arai D, Ishioka K, Ohgino K, Ikemura S, Sato T, et al: Activation of the FGF2-FGFR1 autocrine pathway: A novel mechanism of acquired resistance to gefitinib in NSCLC. Mol Cancer Res 11: 759-767, 2013

12. Nukaga S, Yasuda H, Tsuchihara K, Hamamoto J, Masuzawa K, Kawada I, Naoki K, Matsumoto S, Mimaki S, Ikemura S, et al: Amplification of EGFR wild type alleles in non-small cell lung cancer cells confers acquired resistance to mutation-selective EGFR tyrosine kinase inhibitors. Cancer Res 15: 2078-2089,2017.

13. Kobayashi S, Boggon TJ, Dayaram T, Jänne PA, Kocher O, Meyerson M, Johnson BE, Eck MJ, Tenen DG and Halmos B: EGFR mutation and resistance of non-small-cell lung cancer to gefitinib. N Engl J Med 352: 786-792, 2005.

14. Pao W, Miller VA, Politi KA, Riely GJ, Somwar R, Zakowski MF, Kris MG and Varmus H: Acquired resistance of lung adenocarcinomas to gefitinib or erlotinib is associated with a second mutation in the EGFR kinase domain. PLoS Med 2: e73, 2005.

15. Bean J, Brennan C, Shih JY, Riely G, Viale A, Wang L, Chitale D, Motoi N, Szoke J, Broderick S, et al: MET amplification occurs with or without T790M mutations in EGFR mutant lung tumors with acquired resistance to gefitinib or erlotinib. Proc Natl Acad Sci USA 104: 20932-20937, 2007.

16. Nakachi I, Naoki K, Soejima K, Kawada I, Watanabe H, Yasuda H, Nakayama S, Yoda S, Satomi R, Ikemura S, et al: The combination of multiple receptor tyrosine kinase inhibitor and mammalian target of rapamycin inhibitor overcomes erlotinib resistance in lung cancer cell lines through c-Met inhibition. Mol Cancer Res 8: 1142-1151, 2010.

17. Yano S, Wang W, Li Q, Matsumoto K, Sakurama H, Nakamura T, Ogino H, Kakiuchi S, Hanibuchi M, Nishioka Y, et al: Hepatocyte growth factor induces gefitinib resistance of lung adenocarcinoma with epidermal growth factor receptor-activating mutations. Cancer Res 68: 9479-9487, 2008.

18. Thomson S, Petti F, Sujka-Kwok I, Epstein D and Haley JD: Kinase switching in mesenchymal-like non-small cell lung cancer lines contributes to EGFR inhibitor resistance through pathway redundancy. Clin Exp Metastasis 25: 843-854, 2008

19. Sequist LV, Waltman BA, Dias-Santagata D, Digumarthy S, Turke AB, Fidias P, Bergethon K, Shaw AT, Gettinger S, Cosper AK, et al: Genotypic and histological evolution of lung cancers acquiring resistance to EGFR inhibitors. Sci Transl Med 3: 75ra26, 2011.

20. Gentzler RD and Johnson ML: Complex decisions for first-line and maintenance treatment of advanced wild-type non-small cell lung cancer. Oncologist 20: 299-306, 2015.

21. Butts CA, Ding K, Seymour L, Twumasi-Ankrah P, Graham B, Gandara D, Johnson DH, Kesler KA, Green M, Vincent M, et al: Randomized phase III trial of vinorelbine plus cisplatin compared with observation in completely resected stage IB and II non-small-cell lung cancer: Updated survival analysis of JBR-10. J Clin Oncol 28: 29-34, 2010.

22. Scagliotti GV, Parikh P, von Pawel J, Biesma B, Vansteenkiste J, Manegold C, Serwatowski P, Gatzemeier U, Digumarti R, Zukin M, et al: Phase III study comparing cisplatin plus gemcitabine with cisplatin plus pemetrexed in chemotherapy-naive patients with advanced-stage non-small-cell lung cancer. J Clin Oncol 26: 3543-3551, 2008.
23. Socinski MA, Bondarenko I, Karaseva NA, Makhson AM, Vynnychenko I, Okamoto I, Hon JK, Hirsh V, Bhar P, Zhang H, et al: Weekly nab-paclitaxel in combination with carboplatin versus solvent-based paclitaxel plus carboplatin as first-line therapy in patients with advanced non-small-cell lung cancer: Final results of a phase III trial. J Clin Oncol 30: 2055-2062, 2012.

24. Langer CJ: The emerging world role of irinotecan in lung cancer Oncology (Williston Park) 15 (7 Suppl 8): S15-S21, 2001.

25. Fossella FV: Docetaxel in second-line treatment of non-small-cell lung cancer. Clin Lung Cancer 3 (Suppl 2): S23-S28, 2002.

26. Fletcher JI, Haber M, Henderson MJ and Norris MD: ABC transporters in cancer: More than just drug efflux pumps. Nat Rev Cancer 10: 147-156, 2010.

27. Livak KJ and Schmittgen TD: Analysis of relative gene expression data using real-time quantitative PCR and the 2(-Delta Delta C(T)) Method. Methods 25: 402-408, 2001.

28. Arao T, Fukumoto H, Takeda M, Tamura T, Saijo N and Nishio K: Small in-frame deletion in the epidermal growth factor receptor as a target for ZD6474. Cancer Res 64: 9101-9104, 2004.

29. Yasuda H, Park E, Yun CH, Sng NJ, Lucena-Araujo AR, Yeo WL, Huberman MS, Cohen DW, Nakayama S, Ishioka K, et al: Structural, biochemical, and clinical characterization of epidermal growth factor receptor (EGFR) exon 20 insertion mutations in lung cancer. Sci Transl Med 5: 216ra177, 2013.

30. Busacca S, Sheaff M, Arthur K, Gray SG, O'Byrne KJ, Richard DJ, Soltermann A, Opitz I, Pass H, Harkin DP, et al: BRCA1 is an essential mediator of vinorelbine-induced apoptosis in mesothelioma. J Pathol 227: 200-208, 2012.

31. Cheung-Ong K, Giaever G and Nislow C: DNA-damaging agents in cancer chemotherapy: Serendipity and chemical biology. Chem Biol 20: 648-659, 2013.

32. Haimeur A, Conseil G, Deeley RG and Cole SP: The MRP-related and BCRP/ABCG2 multidrug resistance proteins: Biology, substrate specificity and regulation. Curr Drug Metab 5: 21-53, 2004.

33. Bessho Y, Oguri T, Ozasa H, Uemura T, Sakamoto H, Miyazaki M, Maeno K, Sato S and Ueda R: ABCC10/MRP7 is associated with vinorelbine resistance in non-small cell lung cancer. Oncol Rep 21: 263-268, 2009.

34. Oguri T, Achiwa H, Sato S, Bessho Y, Takano Y, Miyazaki M, Muramatsu H, Maeda H, Niimi T and Ueda R: The determinants of sensitivity and acquired resistance to gemcitabine differ in non-small cell lung cancer: A role of ABCC5 in gemcitabine sensitivity. Mol Cancer Ther 5: 1800-1806, 2006.

35. Gan PP, Pasquier E and Kavallaris M: Class III beta-tubulin mediates sensitivity to chemotherapeutic drugs in non small cell lung cancer. Cancer Res 67: 9356-9363, 2007.

36. Stuckler D, Singhal J, Singhal SS, Yadav S, Awasthi YC and Awasthi S: RLIP76 transports vinorelbine and mediates drug resistance in non-small cell lung cancer. Cancer Res 65: 991-998, 2005.

37. Mackey JR, Mani RS, Selner M, Mowles D, Young JD, Belt JA, Crawford CR and Cass CE: Functional nucleoside transporters are required for gemcitabine influx and manifestation of toxicity in cancer cell lines. Cancer Res 58: 4349-4357, 1998.

38. Goan YG, Zhou B, Hu E, Mi S and Yen Y: Overexpression of ribonucleotide reductase as a mechanism of resistance to 2,2-difluorodeoxycytidine in the human KB cancer cell line. Cancer Res 59: 4204-4207, 1999. 